\title{
Difficulties of Distal Catheter Insertion of Ventriculoatrial Shunting in Infants and Little Children
}

\author{
Hakan ERDOGAN ${ }^{1}$, Adnan ALTUN²${ }^{2}$, Enis KURUOGLU ${ }^{3}$, Ahmet Hilmi KAYA ${ }^{1,4}$, Adnan DAGCINAR ${ }^{5}$ \\ ${ }^{1}$ Adatip Hospital, Neurosurgery Clinic, Istanbul, Turkey \\ ${ }^{2}$ KTO Karatay University, Department of Neurosurgery, Konya, Turkey \\ ${ }^{3}$ Ondokuz Mayis University, Department of Neurosurgery, Samsun, Turkey \\ ${ }^{4}$ Gelisim University, Department of Health Sciences, Istanbul, Turkey \\ ${ }^{5}$ Marmara University, Department of Neurosurgery, Istanbul, Turkey
}

\section{ABSTRACT}

AIM: Ventriculoatrial (VA) shunting is a well-described cerebrospinal fluid diversion method for the treatment of hydrocephalus. However, it may be very challenging in infants and little children because of atrial catheter placement difficulties. This study aimed to create an algorithm to solve problems faced during open surgical procedures based on the present authors' experience.

MATERIAL and METHODS: We conducted a retrospective analysis on 18 infants and children who underwent VA shunt insertion at the Department of Neurosurgery, Ondokuz Mayis University School of Medicine Hospital between 2005 and 2012. Complications, clinical outcomes, revisions, and solutions for overcoming distal catheter placement difficulties were evaluated.

RESULTS: Twenty-six VA shunt operations were performed in 18 patients. Six patients required eight VA shunt revisions. VA shunting was primarily performed from the internal jugular, facial, cephalic, and subclavian veins to the right atrium. In revision procedures, the internal jugular, cephalic, and subclavian veins were used.

CONCLUSION: VA shunting in infants and little children requires careful surgical techniques. Neurosurgeons should necessarily have an appropriate strategy for VA shunting considering the complications and revisions. Our results suggest open surgical solutions to overcome distal catheter placement difficulties in this age group.

KEYWORDS: Difficulty, Distal catheter, Hydrocephalus, Ventriculoatrial shunt

\section{INTRODUCTION}

$\mathrm{V}$ arious sites, including the atrium, pleural cavity, ureter, fallopian tubes, bladder, and gastric lumen, have been defined for the distal catheter implantation of cerebrospinal fluid (CSF) shunts (15). Ventriculoatrial (VA) shunting was commonly used in the surgical management of hydrocephalus (13). However, it has become a second-line option since the peritoneal cavity remains the preferred site for distal catheter placement. Moreover, VA shunting appears to be difficult to perform and prone to get seriously complicated (16). Currently, the primary considerations include peritoneum- related problems such as peritonitis, intra-abdominal pathologies, scarring due to prior procedures, peritoneal septations, ascites and peritoneal absorption failure.

Even though there are fewer indications for VA shunts in daily practice than those of ventriculoperitoneal (VP) shunts, application difficulties and complications should be known. Appropriate surgical techniques can limit the risk of postoperative complications. The insertion of an atrial catheter in infants and small children can be challenging, particularly in revision cases. 
Erdogan H. et al: Difficulties in Ventriculoatrial Shunting

In this study, a retrospective analysis of 18 pediatric patients who underwent VA shunt insertions was conducted, and VA shunt insertion procedure and cardiac catheterization-related problems are discussed.

\section{MATERIAL and METHODS}

This retrospective study was conducted at the Department of Neurosurgery, Ondokuz Mayis University School of Medicine Hospital. Eighteen infants and children who underwent VA shunt insertions were identified, and their medical records were studied. All patients were operated by the senior author from 2005 to 2012 at the same institution. Follow-up was performed at 2 months and 2 years. Reasons of intraperitoneal placement failure, revision-related conditions and techniques were discussed in the light of the foregoing findings.

\section{RESULTS}

The mean age of the patients at the time of VA shunt placement was 30.2 months (range 3 to 60 months). Of all the patients, 10 (55.5\%) were male and eight (44.4\%) were female. The VP shunt was previously used to treat hydrocephalus in all the patients. The causes of hydrocephalus included myelomeningocele $(n=6,33.3 \%)$, tumor $(n=4,22.2 \%)$, meningitis $(n=3,16.6 \%)$, congenital hydrocephalus $(n=2$, $11.1 \%)$, intraventricular hemorrhage due to prematurity $(n=2$, $11.1 \%)$, and hemophagocytic syndrome $(n=1,5.5 \%)$. Before VA shunt placement, 50 operations were performed including VP shunt implantation, VP shunt revision, external ventricular drainage, endoscopic third ventriculostomy and endoscopic fenestration.

Impaired absorptive ability of the peritoneum $(n=14,77.7 \%)$ and intra-abdominal infection $(n=4,22.2 \%)$ were the main causes of peritoneal catheter failure, leading to the necessity of VA shunting.

VA shunting was primarily performed directly via the internal jugular vein in nine patients, via the facial vein (or another vein draining into the internal jugular vein) in six patients, and via the cephalic vein, through the subclavian vein, and directly into the right atrium in three patients. Of these three patients, one was previously operated at another center and two underwent tracheostomy. The cephalic vein was chosen to make a more distant incision to the tracheostomy in two patients, and the approach from the same incision was not safe because of the existing scar from the previous operation in one patient.

Six patients required eight VA shunt revisions (two of them had repeated failure). The most common reason for revision was infection $(n=4,50 \%)$, followed by intra-atrial thrombus $(n=3,37.5 \%)$ and distal catheter disconnection $(n=1,12.5 \%)$.

In a patient, in whom the distal tip of the catheter was disconnected and slipped down into the right ventricle, the detached component was encaged and removed during angiography and VA shunt replacement was performed.

Among the patients who underwent revision operations, two with catheter placement in the facial vein required revision with the internal jugular vein first, followed by the cephalic vein. Two patients with placement in the internal jugular vein required revision with the cephalic vein. In two patients, the subclavian vein was used in the revision by the Seldinger technique where their catheters were previously inserted.

In the 2-year follow-up period, additional VA shunt-related morbidity and mortality were not observed.

\section{DISCUSSION}

VA shunt became popular after the introduction of the Spitz-Holter valve in the early 1950s (13). However, after the recognition of serious complications and operative difficulties, VA shunts were generally avoided (6). A decade later, developed techniques for distal catheter placement in the peritoneal space provided a feasible surgical strategy for the CSF diversion procedure (2). Consequently, starting in the late 1970s, VA shunts became outdated following the introduction of VP shunt.

VA shunt is still a preferable alternative when the peritoneum is not conducive for absorption. VA shunt implantation is also a treatment option when the VP shunt malfunctions because of physiological situations such as enlarging uterus that increases the intra-abdominal pressure during pregnancy (4). Despite the common belief that correct VA shunt placement is difficult and the complication rates are high, there are case series of adults suggesting that VA shunting is as safe as VP shunting $(1,9)$. Certainly, these favored outcomes could be associated with the age distribution of the patients.

It is certainly important to place the shunt through an appropriate vein into the atrium. Recently, several techniques have been described to reduce complications and achieve greater success in atrial catheter replacement. Gonzalez et al. have reported an endovascular technique that utilizes an endoscope-assisted approach (5). Ultrasonographyguided VA shunting has been described $(3,17,18)$, and it was suggested that it could reduce complications by providing the correct placement of the cardiac end of the catheter and allow an X-ray-free procedure (4). Particularly in revisions, percutaneous placement of VA shunt under ultrasonographic guidance using the Seldinger technique would be relatively effective and safe (10). Momin et al. have reported the technique of intraoperative venography to optimize distal catheter placement (11). Moreover, endovascular balloon dilatation could be used to place the catheter when the vein is severely narrowed (12). These methods would also be effective for patients who require VA shunt revision, but it is still necessary to have experience in performing open surgical techniques in VA shunt implantation and revisions. However, we suppose that these techniques are generally used in adults or bigger children and may not be easily used in infants and toddlers whose veins are not large enough for percutaneous or angiographic approaches.

In infants and toddlers, it is usually impossible to easily perform central catheterization even under general anesthesia. Thus, it is mandatory to know about open surgical techniques and potential problems. All the 18 cases were operated by the same surgeon, and complications both during the surgery 
and in the postoperative period led to the discussion of distal catheter placement difficulties and possible solutions. The conventional surgical procedure involves placing the distal catheter into the atrium via the facial or internal jugular vein as described many times in literature (Figure 1).

In little children, different approaches should be applied when the lumen of the facial vein is very narrow and the catheter cannot be introduced through. Another problem is the collapse of the previously used vein and the impossibility of re-catheterization. In such situations, the same incision could be deepened to expose the internal jugular vein in the neurovascular bundle. Venous cut-down can be performed in any vein that drains into the internal jugular vein just before the

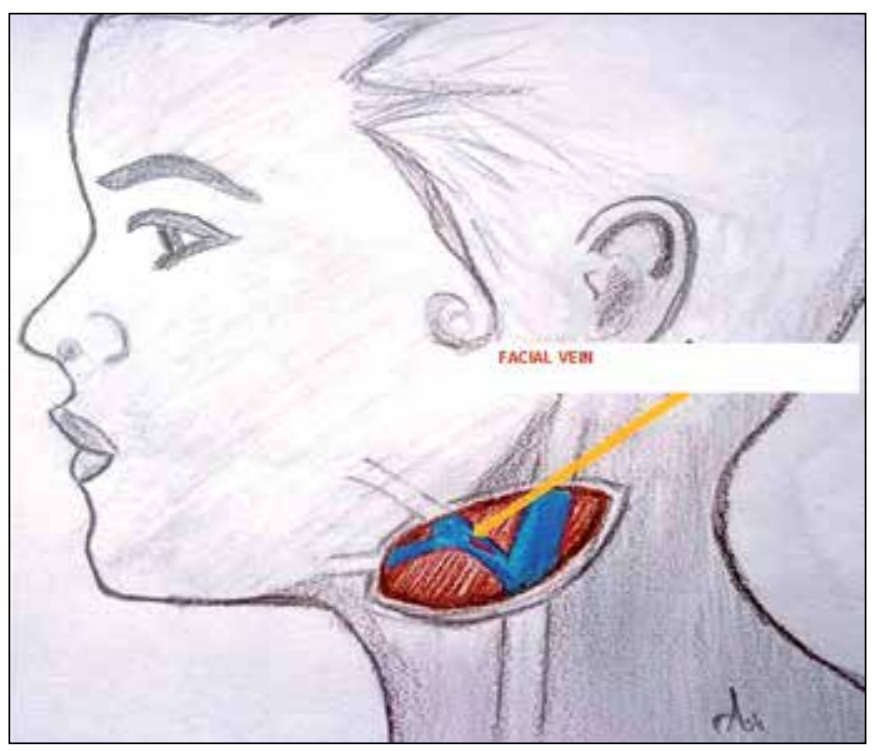

Figure 1: Identification of facial vein for introduction of atrial catheter. drainage point or a catheter could be inserted by venotomy on the internal jugular vein.

The replacement of distal shunt catheters can be extremely hard in revisions, particularly in young patients. Multiple revisions can inevitably cause scarring and thrombosis. If the shunt requires removal, replacement should be done in the same operation (except for infectious complications). Otherwise, limited venous access will be a problem because the veins (facial or internal jugular vein) where the distal catheter is inserted could collapse and cause revision problems.

In revision cases, if the vein was previously used, it is very hard to expose this vein and reintroduce the catheter. In our cases, it was always found to be occluded, and the catheter was implanted from the cephalic or subclavian vein (Figure 2). When we decide to use the cephalic vein, we make an incision that crosses the projection of the deltopectoral sulcus just beneath the clavicle; we then deepen the incision through the pectoral muscle fibers to reach the cephalic vein (Figure 3 ). After exposing the vein, we perform cut-down to the cephalic vein and introduce the catheter using a J-tip guide wire to the right atrium via the subclavian vein. If the facial or cephalic vein was previously used, catheterization of the subclavian vein through a previous subclavicular incision or a new incision connecting the cervical incision using the Seldinger technique may be another option. The left internal jugular vein should not be used during revisions because of the risk of bilateral venous obstruction.

According to several reports that compare VA shunts with VP shunts in pediatric patients, VA shunts require more revisions $(7,8,14)$. In fact, the gradual retraction of the atrial catheter is unavoidable due to the longitudinal growth of children. Thus, children with VA shunts are inherently likely to require revisions (16). In these cases, it is better to replace the longer distal catheter into the atrium through the previously used entrance to the vein. For this reason, using an open-ended catheter in

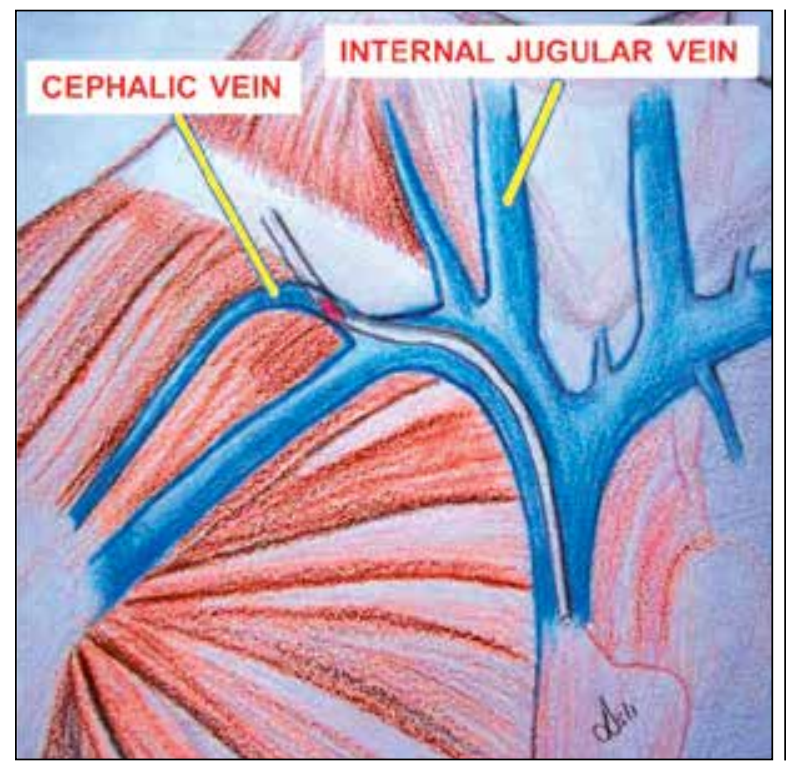

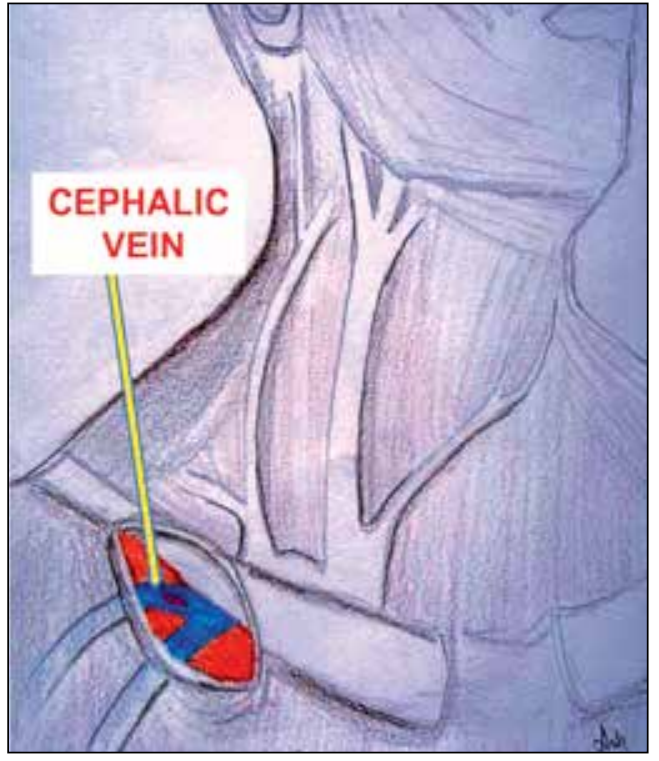

Figures 2 (left) and 3 (right): Identification cephalic vein for introduction of atrial catheter. 


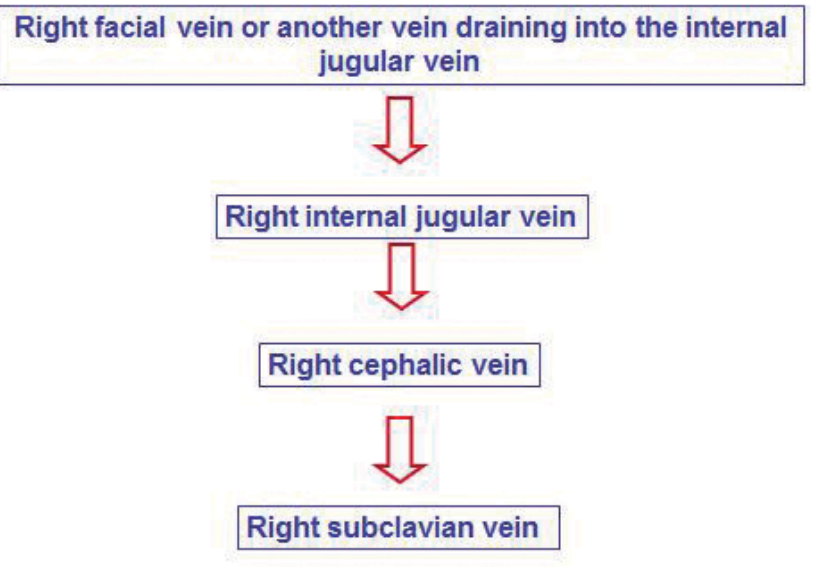

Figure 4: Algorithm for implantation of distal catheter for VA shunting.

the first operation can be suggested. In revision cases, the guide wire can be passed through and lead the new catheter to atrium with ease. Otherwise, the catheterization could be very difficult or impossible.

One of the main points for VA shunt revisions is protection and sparing use of the internal jugular vein and its major tributaries. According to our experience and intraoperative findings, atrial catheter replacement can be very difficult, particularly in revisions. Therefore, we propose an algorithm for selecting the right venous access (Figure 4).

\section{CONCLUSION}

Despite the life-threatening complications, VA shunts are indispensable for CSF drainage in hydrocephalus. The risk of shunt failure and revision-related problems for VA catheterization are of significant concern in infants. However, better results can be obtained by adequate surgical technique and on-site decisions.

\section{ACKNOWLEDGMENT}

The authors wish to thank to Mrs. Aslı Altun for her illustrations.

\section{- REFERENCES}

1. Akthar N, Khan AZ, Yousaf M: Experience and outcome of Ventricular-atrial shunt: A multi centre study. J Ayub Med Coll Abbottabad 27(4):817-820, 2015

2. Ames RH: Ventriculo-peritoneal shunts in the management of hydrocephalus. J Neurosurg 27(6):525-529, 1967

3. Bosi G, Zorzi E, Guerrini P, Lintermans JP: Intraoperative echocardiographic control during ventriculoatrial shunt placement. J Neurosurg 62:783-784, 1985
4. Di Muro L, Pallini R, Pietrini D, Colizzi C, Denaro L: Minimally invasive echo-guided placement of the cardiac tube in a ventriculoatrial shunt during pregnancy: Technical note. Neurosurgery 61(5 Suppl 2): E398, 2007

5. Gonzalez LF, Kim L, Rekate HL, McDougall CG, Albuquerque FC: Endovascular placement of ventriculoatrial shunt. J Neurosurg 106 Suppl 4:319-321, 2007

6. Ignelzi RJ, Kirsch WM: Follow-up analysis of ventriculoperitoneal and ventriculoatrial shunts for hydrocephalus. J Neurosurg 42:679-682, 1975

7. KeucherTR, Mealey J Jr: Long-term results after ventriculoatrial and ventriculoperitoneal shunting for infantile hydrocephalus. J Neurosurg 50:179-186, 1979

8. Little JR, Rhoton AL Jr, Mellinger JF: Comparison of ventriculoperitoneal and ventriculoatrial shunts for hydrocephalus in children. Mayo Clin Proc 47:396-401, 1972

9. McGovern RA, Kelly KM, Chan AK, Morrissey NJ, McKhann GM 2nd: Should ventriculoatrial shunting be the procedure of choice for normal-pressure hydrocephalus? J Neurosurg 120:1458-1464, 2014

10. Metellus P, Hsu W, Kharkar S, Kapoor S, Scott W, Rigamonti $D$ : Accuracy of percutaneous placement of a ventriculoatrial shunt under ultrasonography guidance: A retrospective study at a single institution. J Neurosurg 110:867-870, 2009

11. Momin EN, Recinos PF, Coon AL, Rigamonti D: Use of intraoperative venography to guide the distal portion of a ventriculoatrial shunt past an obstruction in the central veins: Technical case report. Neurosurgery 66 (Suppl Operative 6):370-371, 2010

12. Natarajan A, Mazhar S: Right heart complications of ventriculoatrial shunts. Eur Heart J 32(17):2134, 2011

13. Nulsen FE, Spitz EB: Treatment of hydrocephalus by direct shunt from ventricle to jugular vein. Surg Forum 399-403, 1951

14. Olsen L, Frykberg T: Complications in the treatment of hydrocephalus in children. A comparison of ventriculoatrial and ventriculoperitoneal shunts in a 20 -year material. Acta Paediatr Scand 72:385-390, 1983

15. Pal K, Jindal V: Ventriculo cholecystic shunt in the management of hydrocephalus. Indian Pediatr 44(6):435-437, 2007

16. Paulsen $\mathrm{AH}$, Lundar $\mathrm{T}$, Lindegaard $\mathrm{F}$ : Pediatric hydrocephalus: 40-year outcomes in 128 hydrocephalic patients treated with shunts during childhood. Assessment of surgical outcome, work participation, and health-related quality of life. J Neurosurg Pediatr 16:633-641, 2015

17. Soyeur D, Born J, Lenelle J, Stevenaert A: Two-dimensional echographic localization of intracardiac cerebrospinal fluid shunt catheters. Neurosurgery 14:2-7, 1984

18. Szczerbicki MR, Michalak M: Echocardiographic placement of cardiac tube in ventriculoatrial shunt. Technical note. J Neurosurg 85:723-724, 1996 